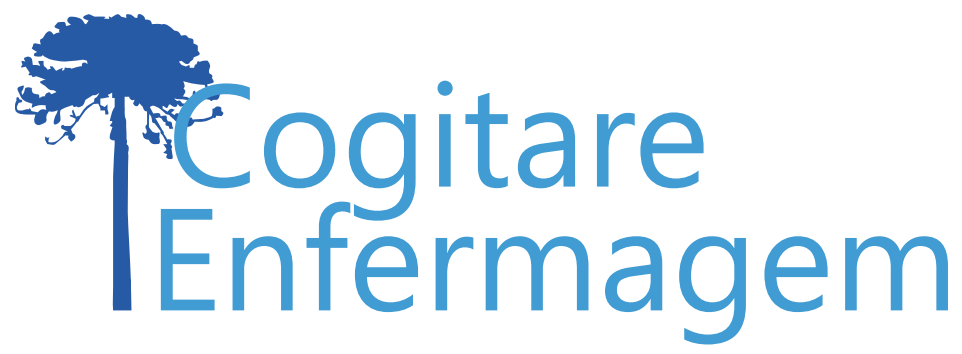

\title{
DIREITOS HUMANOS DAS MULHERES NO PARTO FRENTE À PANDEMIA DE COVID-19: O QUEFAZER DA ENFERMAGEM OBSTÉTRICA
}

Kleyde Ventura de Souza1 ${ }^{1}$ Søndre Schneck², Érica Dumont Pena ${ }^{3}$, Elysângela Dittz Duarte ${ }^{4}$, Valdecyr Herdy Alves ${ }^{5}$

\section{RESUMO}

Objetivo: esta comunicação tem por objetivo provocar a reflexão de enfermeiras(os) obstétricas(os) a seguir na luta pela garantia de direitos das mulheres e de seus bebês, em meio à pandemia de COVID-19.

Desenvolvimento: chama a atenção a violação dos Direitos Humanos das mulheres, seus filhos e de enfermeiras obstétricas e obstetrizes, principalmente pelo impacto da pandemia. Endossa a Declaração da International Confederation of the Midwives intitulada "Os direitos das mulheres no parto devem ser mantidos durante a pandemia", e evoca a Enfermagem Obstétrica a um quefazer, solidário e pautado pela justiça social, construído e anunciado pelos corpos e vozes daquelas(es) que cuidam e são cuidadas(os).

Considerações Finais: sinalizamos o compromisso político-social com a democracia enquanto processo que legitima a cidadania num campo especial de interesse: a vida e a saúde das mulheres, a partir do direito constitucional e dos desafios éticos que nos convoca a pandemia.

DESCRITORES: Direitos Humanos; Infecções por Coronavírus; Enfermagem Obstétrica; Direitos da Mulher; Sistema Único de Saúde.

COMO REFERENCIAR ESTE ARTIGO:

Souza KV de, Schneck SC, Pena ED, Duarte ED, Alves VH. Direitos humanos das mulheres no parto frente à pandemia de Covid-19: o quefazer da enfermagem obstétrica. Cogitare enferm. [Internet]. 2020 [acesso em "colocar data de acesso, dia, mês abreviado e ano"]; 25. Disponível em: http://dx.doi.org/10.5380/ce.v25i0.73148.

Este obra está licenciado com uma Licença Creative Commons Atribuição 4.0 Internacional.

${ }^{1}$ Enfermeira Obstétrica. Doutora em Enfermagem. Docente de Enfermagem da Universidade Federal de Minas Gerais. Presidente da Associação Brasileira de Obstetrizes e Enfermeiros Obstetras - Nacional. Belo Horizonte, MG, Brasil. $\bigcirc$

${ }^{2}$ Enfermeiro Obstétrico. Doutor em Ciências. Docente de Enfermagem da Universidade Federal do Rio Grande do Sul. Porto Alegre, RS, Brasil. 9

${ }^{3}$ Enfermeira. Doutora em Educação. Docente de Enfermagem da Universidade Federal de Minas Gerais. Belo Horizonte, MG, Brasil. 1

${ }^{4}$ Enfermeira. Doutora em Saúde da Criança e do Adolescente. Docente de Enfermagem da Universidade Federal de Minas Gerais. Belo Horizonte, MG, Brasil. $\bigcirc$

${ }^{5}$ Enfermeiro Obstétrico. Doutor em Enfermagem. Docente da Universidade Federal Fluminense. Niterói, RJ, Brasil. 9 


\title{
HUMAN RIGHTS OF WOMEN IN CHILDBIRTH IN THE CONTEXT OF THE COVID-19 PANDEMIC: WHAT OBSTETRIC NURSING HAS TO DO
}

\begin{abstract}
Objective: This release aims to promote the reflection of obstetric nurses so that they continue to fight to guarantee the rights of women and their babies amidst the COVID-19 pandemic. Development: This paper draws the attention to the violation of the Human Rights of women, their children, and obstetric nurses and midwives, mainly due to the impact of the pandemic. It endorses the Official Statement of the International Confederation of the Midwives named "Women's Rights in Childbirth Must be Upheld During the Coronavirus Pandemic", and evokes Obstetric Nursing to a solidary work guided by social justice, built and announced by the bodies and voices of those who provide care and those who receive it.

Final Considerations: We signal the political and social commitment to democracy as a process that legitimizes citizenship in a special field of interest: women's life and health, based on the constitutional law and on the ethical challenges that are imposed by the pandemic.
\end{abstract}

DESCRIPTORS: Human Rights; Infections by Coronavirus; Obstetric Nursing; Women's Rights; Unified Health System.

\section{DERECHOS HUMANOS DE LAS MUJERES EN EL PARTO FRENTE A LA PANDEMIA DE, COVID-19: LO QUE SE DEBE HACER EN ENFERMERIA OBSTÉTRICA}

\begin{abstract}
RESUMEN:
Objetivo: este comunicado pretende provocar la reflexión de los profesionales de Enfermería obstétrica para que prosigan en la lucha por la garantía de los derechos de las mujeres y de sus bebés, en plena pandemia de COVID-19.

Desarrollo: concentra la atención en la violación de los Derechos Humanos de las mujeres, de sus hijos y de las enfermeras obstétricas y obstetras, principalmente por el efecto de la pandemia. Apoya la Declaración de la International Confederation of the Midwives llamada "Los derechos de las mujeres en el parto deben mantenerse durante la pandemia", y evoca a la Enfermería obstétrica a un comportamiento solidario y pautado por la justicia social, construido y anunciado por los cuerpos y las voces tanto de quienes prestan servicios de cuidado como de quienes los reciben.

Consideraciones finales: señalamos el compromiso político-social con la democracia con respecto al proceso que legitima a la ciudadanía en un campo especial de interés: la vida y la salud de las mujeres, a partir del derecho constitucional y de los desafíos éticos que nos impone la pandemia.
\end{abstract}

DESCRIPTORES: Derechos Humanos; Infecciones por Coronavirus; Enfermería obstétrica; Derechos de la mujer; Sistema Único de Salud. 
No ano de 2020, Ano Internacional de Enfermeiras e Obstetrizes, declarado pela Organização Mundial de Saúde (OMS) e amplamente divulgado na Campanha Nursing Now, que iniciamos em meio à grave pandemia de COVID-19, a presente comunicação pretende ser uma provocação. Provocar, no sentido que a palavra se constitui: pro (adiante) e vocar (chamar). Sinalizamos, portanto, o compromisso político-social com a democracia enquanto processo que legitima a cidadania num campo especial de interesse: a vida e saúde das mulheres, a partir do direito constitucional e dos desafios éticos que nos convoca a pandemia.

Como acadêmicas(os), pesquisadoras(es), formadoras(es) da força de trabalho na Enfermagem/Enfermagem Obstétrica no âmbito da graduação e pós-graduação, trabalhadoras(es) da linha de frente em maternidades e representantes da Enfermagem na área de saúde das mulheres, mais especificamente no campo obstétrico neonatal provocamos leitoras e leitores a refletir sobre a necessidade de garantia e potencialização dos direitos das mulheres, bem como a promoção dos melhores níveis de atenção à sua saúde e de seus filhos.

Especialmente neste tempo de emergência sanitária, decorrente da pandemia da COVID-19, convidamos a todos para ir adiante na garantia desses direitos, sem recuar nenhum passo. Chamamos hoje a caminhar em direção a um futuro incerto, porém com a única certeza de que não se pode deixar ninguém para trás.

\section{DESENVOLVIMENTO}

No campo do parto e nascimento, a violação dos direitos humanos das mulheres, de seus filhos e também de enfermeiras obstétricas e obstetrizes, principalmente pelo impacto da pandemia de COVID-19 em suas vidas, impõe sua defesa, como enfatiza a Declaração da International Confederation of the Midwives (ICM) intitulada "Os direitos das mulheres no parto devem ser mantidos durante a pandemia"(1). Esses direitos se constituem no campo dos Direitos Humanos. A declaração é um marco referencial para que a resposta à pandemia garanta o atendimento às necessidades de mulheres e bebês, num momento singular de suas vidas, o parto e o nascimento; mas também a outros direitos reprodutivos.

Endossamos a Declaração da ICM - entidade que representa e trabalha para fortalecer associações profissionais de enfermeiras obstétricas e obstetrizes em todo mundo. Alinhada à $\mathrm{OMS}$ e ao Fundo de População das Nações Unidas (UNFPA), pela ressonância com os marcos da Associação Brasileira de Obstetrizes e Enfermeiros Obstetras (ABENFO Nacional) (2), relacionados à defesa da vida com dignidade e bem-estar, além do fortalecimento da profissão e de suas(seus) trabalhadoras(es), como nos convoca a Campanha Nursing Now e como ensejam/fazem aquelas(es) que a constroem e a (re)inventam. Marcos que ganham ainda mais sentido quando nos provocam a Enfermagem Obstétrica a um quefazer. Tudo isso no âmbito do maior sistema de saúde do mundo, o Sistema Único de Saúde (SUS) do Brasil, de modo que o "agora e sempre" da Enfermagem e da Obstetrícia se revele de forma ainda mais potente em 2020, diante do desafio de colaborar incondicionalmente com resposta à pandemia de COVID-19.

Nesse sentido, reafirmamos que todas as mulheres têm o direito de receber atenção qualificada em uma rede de atenção à saúde, cujos arranjos devem garantir o acesso, considerando a integralidade e a humanização do cuidado. Isto inclui fluxos de atendimento específicos às gestantes, que as protejam em locais mais seguros, priorizando centros de partos normais (CPN) e maternidades, nos casos de baixo risco. Tudo isso deve ser observado com atenção máxima considerando o cenário de alta complexidade da pandemia COVID-19, que exige as melhores respostas e nos convoca a uma ação coordenada efetiva 
e sensível às necessidades de todos.

Nos últimos 30 anos, a criação do SUS e a implementação de políticas públicas concretizadoras de direitos sociais, orientadas pela Constituição de 1988, devem ser apontadas como aspectos fundamentais do aprimoramento da atenção no campo da saúde das mulheres, bem como os desafios que ainda persistem: as mortes evitáveis de mulheres, a lenta queda da razão de mortalidade materna, a criminalização do aborto, as altas taxas de cesarianas, a violência obstétrica, a necessidade de superar o modelo medicalizado na atenção ao parto e nascimento, entre outras (3). A Declaração da ICM enfatiza: "Toda mulher e seu recém-nascido têm direito a tratamento com compaixão, dignidade e respeito"(1:1), nos convocando/provocando a um quefazer.

No contexto da pandemia, as mulheres podem encontrar mais dificuldades para que suas escolhas sejam escutadas, acolhidas e respeitadas pelos serviços e profissionais da saúde, relacionado às limitações que a crise sanitária impõe a todo o sistema. Essas dificuldades encontram na Declaração(1) seu contraponto: "Toda mulher tem o direito à informação, a dar seu consentimento, a negar-se a consentir e que sejam respeitadas e defendidas as suas escolhas. Isto inclui o direito a ter o acompanhante de sua escolha com ela durante o trabalho de parto e nascimento" ${ }^{\prime(1: 1)}$.

É preciso considerar que a crise causada pela pandemia deverá impactar a qualidade da atenção e gerar maior exposição para o aumento de taxas de cesarianas realizadas sem indicação clínica precisa e incremento da violência de gênero, obstétrica e institucional. Tais condições podem repercutir de forma intensa e negativa na experiência reprodutiva das mulheres, na saúde de seus bebês, famílias e comunidade. São consequências de natureza social, estrutural, cultural, emocional, e outras mais amplas, do campo econômico e estrutural, decorrentes das demais implicações da pandemia no conjunto do tecido social, que podem comprometer a experiência positiva de parto, como incentiva a recomendação da OMS intitulada "Intrapartum care for a positive childbirth"(4). Volta à cena a urgente necessidade de correção de rumos em relação às desigualdades de gênero que afetam a vida das mulheres.

Dentre os destaques feitos na Declaração(1), pontuamos alguns que podem ser inscritos numa perspectiva de avanços ainda frágeis, ou porque não foram plenamente alcançados ou porque são passíveis de retrocessos. Por isso, devem ser tomados como alertas: a garantia de acompanhante de escolha da mulher no processo de parto e nascimento, que no Brasil é garantida por lei ${ }^{(5)}$, as consequências negativas de intervenções realizadas de forma inadequada, e o respeito à autonomia das mulheres e sua integridade física e emocional.

Assim, dois pontos da Declaração ganham destaque:

i) intervenções médicas de rotina como a indução do parto, a cesariana e o uso de fórceps sem indicação obstétrica aumentarão a probabilidade de complicações para a mãe e recém-nascido, bem como a duração da internação hospitalar e a carga de trabalho dos profissionais de saúde nos hospitais, todos com maior possibilidade de exposição à COVID-19;

ii) atualmente, não há evidências que sugiram que as mulheres não possam dar à luz por meio de parto vaginal ou que seja mais seguro realizar uma cesariana em caso de suspeita ou confirmação de COVID-19(1:1).

Reitera-se na Declaração(1): "As decisões da mulher em relação ao parto devem ser respeitadas e seguidas o máximo possível, tendo em consideração suas necessidades clínicas" ${ }^{\prime \prime}(1)$.

Nessa perspectiva, o alerta é para os(as) gestores(as), as(aos) trabalhadoras(es), as usuárias e suas famílias, as instâncias de controle social e todos os demais entes comprometidos com a garantia dos direitos das mulheres. É premente a necessidade de se manter a rede de atenção à saúde organizada em seus diversos níveis, visto que é 
constituída por serviços essenciais: a atenção à gestação, parto, pós-parto, aleitamento materno, bem como a saúde reprodutiva - incluindo serviços de atenção ao aborto legal e de atenção humanizada às mulheres vítimas de violência sexual e de outras formas de violência contra a mulher, nos termos da Convenção sobre a Eliminação de Todas as Formas de Discriminação contra a Mulher (CEDAW) e da Convenção Interamericana para Prevenir, Punir e Erradicar a Violência contra a Mulher, de acordo com o disposto na Lei Maria da Penha ${ }^{(6)}$.

É necessário, finalmente, continuar mantendo os olhares para as diversas especificidades das mulheres, sobretudo no Brasil, um país marcado por profundas desigualdade sociais e regionais que se interseccionam com desigualdades de gênero e raça/cor/etnia. Ou seja, mulheres pobres, negras, periféricas, indígenas, em situação prisional, situação de rua, dentre outras condições que se configuram como barreiras para o acesso aos serviços de saúde. Estas mulheres, já em isolamento social, correm maior risco de ter em suas vidas o impacto negativo da pandemia de COVID-19, com consequências no curto, médio e longo prazo.

O racismo e a violência institucional em saúde devem ser continuamente combatidos e eliminados, sobretudo nos momentos de crise sanitária como a que atravessamos. Assim, indicamos que, principalmente em tempo de pandemia do Severe Acute Respiratory Syndrome Coronavirus 2 (SARS-Cov2), o Coronavírus, como enfatiza a Declaração: "A atenção à saúde sexual e reprodutiva, como o planejamento familiar, a anticoncepção de emergência e aos serviços de aborto, devem ser mantidos disponíveis nos serviços de saúde"(1:2).

Sobre esse aspecto, ganha relevância um grande desafio em nosso país: o modelo de cuidado. No Brasil, em consonância com órgão internacionais, como a OMS e o Ministério da Saúde ${ }^{(7)}$, recomenda-se que os gestores de saúde devem proporcionar condições para a implementação de um modelo de atenção que inclua a enfermeira obstétrica e obstetriz na assistência ao parto de mulheres com risco habitual. Também não deve prescindir da atuação dessas profissionais no cuidado às mulheres com condições de risco clínico ou obstétrico em conjunto com a equipe de saúde.

A Declaração enfatiza o modelo de cuidado e chama a atenção para a importância da rede de atenção:

i) modelos de continuidade dos cuidados em Obstetrícia (pelo mesmo profissional) reduzirão o número de cuidadores em contato com a mulher e seu acompanhante e diminuirão as possibilidades de propagação da COVID-19 nos hospitais, deve-se motivar a proporcionar o modelo de continuidade do cuidado em Obstetrícia;

ii) nos países nos quais os sistemas de saúde podem apoiar o parto em ambiente domiciliar, as mulheres saudáveis que têm uma gestação de risco habitual e com o apoio de obstetrizes e enfermeiras obstétricas, em conjunto com a retaguarda de emergência adequada, podem ter um parto seguro no domicílio ou em um centro de parto no nível da atenção primária, ao invés de um hospital no qual pode haver muitos casos de pacientes (inclusive pacientes que não necessitam de cuidados obstétricos) com COVID-19;

iii) a atenção pré-natal deve continuar sendo prioritariamente desenvolvida no âmbito da atenção básica ${ }^{(1: 2)}$.

A Declaração da ICM tem foco específico nas ações direcionadas às mulheres, aos serviços e aos sistemas de saúde, e destaca ainda os aspectos relacionados aos trabalhadores: Enfermeiras(os) Obstétricas(os), Obstetrizes, Enfermeiras (os)s generalistas, Auxiliares e Técnicas(os) de Enfermagem, bem como os demais trabalhadores e pessoal da saúde, tendo em vista a necessidade de garantir todas as condições necessárias para o desempenho, com segurança, das atividades que compõem a resposta à pandemia.

Neste sentido, as entidades da área, nacionais e internacionais, como a ABENFO, o sistema COFEN-CORENs e a própria ICM, ressaltam que todos os profissionais devem ter 
à disposição Equipamento de Proteção Individual (EPI) e que a valorização do trabalho e dos trabalhadores - gente que move/concretiza/inventa o SUS, tomada como dispositivo para inovação e intervenção, potencializando, assim, a reorganização dos processos trabalho e do viver das(os) trabalhadoras(es). Enfermeiras(os) Obstétricas/Obstetras e Obstetrizes, assim como as(os) demais trabalhadoras(es) do setor saúde com atuação na linha de frente na luta contra a pandemia, pelo impacto da crise e por serem afetadas(os) desproporcionalmente pelas questões de gênero, devem ter reconhecidas e garantidas suas necessidades.

\section{CONSIDERAÇÕES FINAIS}

Convocamos a todas(os) para seguir nos rumos norteados pelos princípios da Saúde Global, dos Objetivos de Desenvolvimento Sustentável (ODS) e, no âmbito nacional, pelas políticas públicas e estratégias que foram construídas no contexto do SUS: a Política Nacional de Atenção Integral à Saúde da Mulher; a Política Nacional de Humanização, a Rede Cegonha, e projetos com força para revigorar o SUS, como os de educação profissional em saúde no desafio da formação-intervenção, por atualizar seus princípios, entre outros, o de integração ensino-serviço, a exemplo de recentes iniciativas do Ministério da Saúde para formação de enfermeiras obstétricas em âmbito nacional(8,9) e o Projeto ApiceON (Aprimoramento e Inovação no Cuidado e no Ensino em Obstetrícia e Neonatologia)(10).

Estas ações, todas balizadas nos Direitos Humanos, na equidade de gênero e no enfoque integral, orientadas por parcerias, centradas nas pessoas, nos coletivos (inclusive de trabalhadoras/es) e na comunidade, nos inspiram a caminhar em frente, ao mesmo tempo em que produzimos um quefazer da Enfermagem Obstétrica. Um quefazer solidário e pautado pela justiça social que, pelo momento crítico, deve ser construído em nossos corpos e anunciado pelas nossas vozes. Se nos dirigimos a um futuro ainda incerto, a única certeza é que devemos seguir juntas(os).

\section{REFERÊNCIAS}

1. International Confederation of Midwives (ICM). Women's rights in childbirth must be upheld during the coronavirus pandemic. [Internet]. [acesso em 22 abr 2020]. Disponível em: https://www. internationalmidwives.org/assets/files/news-files/2020/03/icm-statement upholding-womens-rightsduring-covid19-5e83ae2ebfe59.pdf.

2. Souza KV de, Lima ML de, Tyrrell MAR, Santos R da S, Mamede MV, Alves VH. Opening speech at the IX COBEON*, III CIEON* and ABENFO* 2015 em Belém, PA, Brazil. Acta Paul. Enferm. [Internet]. 2016 [acesso em 22 abr 2020]; 29(1). Disponível em: https://doi.org/10.1590/1982-0194201600017.

3. Leal M do C, Szwarcwald CL, Almeida PVB, Aquino EML, Barreto ML, Barros F, et al. Reproductive, maternal, neonatal and child health in the 30 years since the creation of the Unified Health System (SUS). Ciênc. Saude Colet [Internet]. 2018 [acesso em 22 abr 2020]; 23(6). Disponível em: https://doi. org/10.1590/1413-81232018236.03942018.

4. World Health Organization (WHO). WHO recommendations: intrapartum care for a positive childbirth experience. [Internet] Geneva: WHO; 2018. [acesso em 22 abr 2020]. Disponível em: https://www.who.int/ reproductivehealth/publications/intrapartum-care-guidelines/en/.

5. Brasil. Lei n. 11.108, de 7 de abril de 2005. Altera a Lei no 8.080, de 19 de setembro de 1990, para garantir às parturientes o direito à presença de acompanhante durante o trabalho de parto, parto e pósparto imediato, no âmbito do Sistema Único de Saúde [Internet]. 07 abr 2005 [acesso em 02 maio 2020]. Disponível em: http://www.planalto.gov.br/ccivil 03/ Ato2004-2006/2005/Lei/L11108.htm. 
6. Brasil. Lei. 11.340 de 7 de agosto de 2006. Dispõe sobre mecanismos para coibir a violência doméstica e familiar contra a mulher. [Internet] 7 ago 2006 [acesso em 22 abr 2020]. Disponível em: http://www. planalto.gov.br/ccivil 03/ ato2004-2006/2006/lei/l11340.htm.

7. Ministério da Saúde (BR). Secretaria de Ciência, Tecnologia e Insumos Estratégicos. Departamento de Gestão e Incorporação de Tecnologias em Saúde. Diretrizes nacionais de assistência ao parto normal: versão resumida. [Internet]. Brasília: Ministério da Saúde; 2017 [acesso em 22 abr 2020]. Disponível em: http://bvsms.saude.gov.br/bvs/publicacoes/diretrizes nacionais assistencia parto normal.pdf.

8. Souza KV de, Santos Filho SB. Educação profissional em saúde: metodologia e experiências de formação-intervenção-avaliação. Porto Alegre: Moriá; 2020.

9. Santos Filho SB, Souza KV de. Methodology to articulate the process of training-interventionevaluation in the professional education in nursing. Ciênc. Saude Colet. [Internet]. 2020 [acesso em 24 abr 2020]; 25(1). Disponível em: https://doi.org/10.1590/1413-81232020251.28322019.

10. Ministério da Saúde (BR). APICEON: aprimoramento e inovação no cuidado e ensino em obstetrícia e neonatologia. Brasília: Ministério da Saúde; 2017.

Recebido: 24/04/2020

Finalizado: $13 / 05 / 2020$

Editora associada: Luciana Puchalski Kalinke

Autor Correspondente:

Kleyde Ventura de Souza

Universidade Federal de Minas Gerais

Av. Prof. Alfredo Balena, 190 - 30130-100 - Belo Horizonte, MG, Brasil

E-mail: venturakleyde@gmail.com

Contribuição dos autores:

Contribuições substanciais para a concepção ou desenho do estudo; ou a aquisição, análise ou interpretação de dados do estudo - KVS, SCS, EDP

Elaboração e revisão crítica do conteúdo intelectual do estudo - EDD, VHA

Aprovação da versão final do estudo a ser publicado - KVS, SCS

Responsável por todos os aspectos do estudo, assegurando as questões de precisão ou integridade de qualquer parte do estudo - KVS, SCS 